\title{
The Forecasted Research of Failure Analysis And Failure Trend About Electronic Equipments
}

\author{
Gao Xiaozhuang ${ }^{1, \text { a }}$, Ding Youhua ${ }^{2, \mathrm{~b}}$,Zhang Peng; ${ }^{3, \mathrm{c}}$ \\ ${ }^{1}$ Department of Radar, Wuhan Mechanical College, Wuhan,430075 ,China \\ ${ }^{2}$ Department of Radar, Wuhan Mechanical College, Wuhan,430075,China \\ ${ }^{3}$ Department of Radar, Wuhan Mechanical College, Wuhan,430075,China \\ email:agaoxz900@sohu.com, ${ }^{\mathrm{b}}$ email: Dingyh1010@sohu.com, ${ }^{\mathrm{c}}$ email: bigbird616@sohu.com
}

Keywords: electronic equipments; failure analysis; failure trend; forecasted model

Abstract: The electronic equipments have numerous kinds and complicated configuration, which have been largely used in army. How to upgrade the maintaining ability of electronic have always been our work point. The research aim is to find out the main reason which causing electronic system failures from electronic failure phenomenon and failure development trend. So we can provide reliable and practice-checkout knowledge. The knowledge can be used to diagnose failures, forecast failure trend and design maintaining technique.

\section{Introduction}

Therefore, the process of using the system in fault and repair, alternating the possibility of the existence of some dependent failure data under the effect of fault data, that is not necessarily independent and identical distribution, fault data changes over time show a certain trend.

Statistical analysis of electronic equipment fault method is the use of probability theory and mathematical statistics, has occurred or may occur fault equipment and unit qualitative or quantitative analysis of failure mode and failure mechanism, identification equipment, estimation of its failure mode and effect of equipment to produce and harmfulness, take measures to make the equipment more reliable the work of .

Fault analysis of statistics, including the analysis of micro and macro statistics statistical analysis. Micro statistical analysis this paper mainly discussed for electronic equipment has a large number of equipped in practical teaching and application of a large number of fault data, through the analysis of the fault of large volumes of data, finally obtains the electronic equipment circuit board even components common malfunction mode of mechanism and system, so as to fault diagnosis and maintenance design services.

\section{The characteristics of fault data}

Object fault analysis of micro statistics is the fault data, record data, state monitoring data from the actual exposure of equipments in the usage phase fault recorded data and equipment design and the factory after resume books recorded information.

Research on fault data analysis is the content of system changes with time running performance, from the beginning until failure time reliability and working life, system repair in various fault again after working reliably time (time between failures). For a repairable system, if given at any time, in the 0 to the frequency of faults time system, may achieve $1,2, \ldots$ Equivalent, is a random variable, so is a random process. The failure process of available stochastic point process representation, as shown in Figure 1.[1]

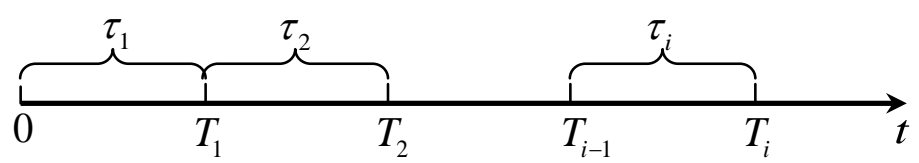

Figure 1: The failure process of repairable system 
$T_{1}<T_{2}<\cdots<T_{i}<\cdots T_{n}<T_{n+1}=t$ is the fault time system, $\tau_{i}=T_{i}-T_{i-1}$ is time between failures

(TBF), which is Fault data.

Without considering the maintenance time case, where $T_{i}$ is the i time the first fault time system, $\tau_{i}$ said $\mathrm{i}-1$ from the time after the repair work to the $\mathrm{m}$ time when the failure of work time, called the adjacent fault interval, so the system has a number of working life.

If the system is able to "repair", and operating conditions are unchanged, adjacent time between failures $\tau_{1}, \tau_{2} \cdots \tau_{n}$ can be thought of as a set of independent identically distributed random variables, so it can be regarded as is on the same system or components are obtained by the test of a life of sample and statistical analysis.

But not all repairable system can be used to describe the "repair", with the operating conditions change, after the repair system working time increased or decreased. There may be the following circumstances:

System failure and maintenance after eliminating the weak links, with the increase in the number of maintenance work time will gradually increase.[2]

With the repair times increase working time will be shortened gradually.

Therefore, the process of using the system, the fault and maintenance of alternating there may be a dependent failure data under the effect of fault data, that is not necessarily independent and identical distribution, fault data changes over time show a certain trend.

According to the point process theory, fault trend available fault process intensity function representation. It is defined as the probability of system failure occurs in a unit of time, i.e.

Therefore, the process of using the system in fault and repair, alternating the possibility of the existence of some dependent failure data under the effect of fault data, that is not necessarily independent and identical distribution, fault data changes over time show a certain trend.

$$
h(t)=\lim _{d t \rightarrow 0} \frac{E(d N(t))}{d t}=\lim _{d t \rightarrow 0} \frac{P([t, t+d t])}{d t}
$$

Therefore, the process of using the system in fault and repair, alternating the possibility of the existence of some dependent failure data under the effect of fault data, that is not necessarily independent and identical distribution, fault data changes over time show a certain trend.

Therefore, the process of using the system in fault and repair, alternating the possibility of the existence of some dependent failure data under the effect of fault data, that is not necessarily independent and identical distribution, fault data changes over time show a certain trend.[3]

Therefore, the process of using the system in fault and repair, alternating the possibility of the existence of some dependent failure data under the effect of fault data, that is not necessarily independent and identical distribution, fault data changes over time show a certain trend.

Among them: $p([t, t+d t])$ is the probability of failure time. $h(t)$ From a certain extent, said the trend and magnitude of repairable system fault occurs over time.

\section{The data analysis 1 based on the reliability mode}

Based on the reliability analysis of model data, mathematical model which is used generally can be divided into two categories: probabilistic models and statistical model. Probability model is from the structure and components of the system, the life distribution of repair time distribution of the relevant information, infer the number associated with the system reliability index of life such as reliability, failure rate, repair rate, can further discuss the optimal design of the system, the use of maintenance strategy; statistical model is starting from the observation the data of parts or units, the life of estimation and test.

Electronic equipment life usually prescribed to failure of the working time for equipment work. The occurrence of the fault is random, therefore, electronic equipment, life is a random variable, the 
mathematical expectation as the average life expectancy. For non repairable component, the average life expectancy is refers to the electronic equipment MTBF; for repairable parts, the average life expectancy of refers to the average working hours MTBF in.[4]

By the random process theory, describing the stochastic process model of repairable system fault data are mainly homogeneous Poisson process model (HPP), model of non homogeneous Poisson process (NHPP), the update process model (RP) and branching Poisson process model (BPP) etc..

With the fault data of homogeneous Poisson process model description, adjacent time $\tau_{1}, \tau_{2} \cdots \tau_{n}$ between failures are independent of each other to follow an exponential distribution. The mean failure interval an arbitrary length $E(N(t))=\lambda_{t}$, is the number in the number of failures per unit time.

Time between failure nonhomogeneous Poisson process description $\tau_{1}, \tau_{2} \cdots \tau_{n}$ is neither independent of each other, but also different distribution in the interval, and the same length, the average number of failure depends not only on the interval length, start still depends on the interval, so non homogeneous Poisson process model is used to describe the non "repair" element.

The update process is generalized homogeneous Poisson process, it can describe the life form of repairable components is more widely.

If a repairable system with different levels of maintenance, every certain period for a complete repair, this only minimal maintenance, fault data independence but different distribution, available at this time branching Poisson process model description.

\section{Analysis of failure data trend}

The method of mathematical statistics based on the fault trend prediction based on fault is the distribution of data analysis. In the influence of fault and repair the system failure, can repair the data changes over time shows some trends or some kind of correlation. Therefore, it is necessary to analyze the tendency of fault data. In practice, to determine a set of adjacent fault interval belongs to a kind of stochastic process, can be carried out through the method of hypothesis testing.[5]

Fault data to analysis of a repairable system, firstly deal with the fault trend test data, to identify whether the data conforms to a trend. If the fault data exist some trends, should also discriminant is monotonic or non monotonic trend trend, and test process of fitting and fitting test of consistency and validity, if the fitting is effective, NHPP model can be used to analyze the fault data; if there is not a trend, should also distinguish fault data whether there is a correlation, if correlation analysis is available, the correlation analysis method or BPP model, if there is no certain correlation, then the fault data are independent and identically distributed, also should be inspected index etc., discrimination is the HPP model or the RP model. So that we can systematically determine the actual changes can repair the system fault data.

In mathematics on the adjacent fault interval time of renewal process model using discriminant test statistic to test whether the adjacent time between failures are independent and identically distributed. If it is, can be turned into statistical problems cannot be repaired components for processing; Weibull process otherwise according to the nonhomogeneous Poisson process for processing.

Given the large number of actual data validation in reliability studies, large and complex electronic distribution system fault repair often in exponential distribution is given priority to, so, in the renewal process model and the homogeneous Poisson process test of fault data, the fitting distribution, can be the first to test whether belongs to the exponential distribution of adjacent time between failures, if not belongs to the exponential distribution, which can go with other distribution fitting. 
Get regularities of distribution of fault data, we can draw the following electronic equipment reliability function and index according to the regularity of distribution.

The reliability of a given time: at a certain point in time, the system can work in the probability of success. For example, some equipment in 2 hours after the probability of successful operation for 0.2 .

The failure probability of a given time: at a certain point in time, the probability of equipment or component failure. The failure probability is also known as the "uncertainty", it and the reliability is opposite.[6]

Life expectancy: components can work in average how much time before failure. Such as the average life span (MTTF) or (MTBF).

Therefore, the process of using the system in fault and repair, alternating the possibility of the existence of some dependent failure data under the effect of fault data, that is not necessarily independent and identical distribution, fault data changes over time show a certain trend.

Loss of efficiency: equipment failure occurred at some time after the probability per unit time.

\section{The development trend of fault analysis and diagnosis of prediction technology}

In recent years, adaptation and fault diagnosis technology based on knowledge in fault analysis, based on the fault and faulty equipment, system, process of detailed analysis and in-depth research, clear the fault generated links, fault propagation way, understand the typical form, typical characteristics and probability of fault mode, the performance of fault, and the combination of analysis on the historical fault data, get the diagnosis prediction and maintenance decision of relevant knowledge, has become the main objective and development trend of fault analysis.

At present, the technology of fault diagnosis knowledge in qualitative knowledge and quantitative treatment which combines qualitative and quantitative knowledge research and development aspects of attention based on. The knowledge of quantization of dealing with imprecise and descriptive by using the fuzzy mathematics method, not only contribute to the enhancement of the reliability of fault reasoning process is rigorous and diagnosis results, but also for using analytical redundancy method and neural network method to create the conditions of knowledge redundancy.[7]

With the expert system, neural network, machine learning, knowledge discovery of intelligent theory and technology gradually mature in the application, from the early 90's, this theory and method to start gradually infiltrated into the built-in test (Built-in-test) technology to form the intelligent BIT technology, the exact definition of the concept of intelligent BIT are as follows: intelligent BIT is including expert system, neural network, fuzzy theory, information fusion, intelligent theory is applied to the BIT design, detection, diagnosis, decision-making, improve the comprehensive efficiency of BIT, the theory, technology and method to reduce the equipment life cycle cost. In electronic system fault analysis, fault monitoring and forecasting, BIT as an important means of fault monitoring and forecasting, is being developed as a comprehensive system in condition monitoring, fault diagnosis, fault isolation, fault prediction and other functions in a body.

\section{Conclusion}

This paper introduces the characteristics of fault data and the common fault of distribution; then on this basis, the analysis of fault data reliability model is made based on the preliminary research, presents several stochastic process model of system failure data recovery, based on the research above, a discriminant discriminant and process method of fault stochastic process model theory of statistical test. Based on the given.

\section{References}

[1] The Research of Distributed Power Quality on-line Monitoring System Based on GRPS[A]. Proceedings 2010 IEEE International Conference on Software Engineering and Service Sciences[C]. 2010 
[2] Implementation and Performance Analysis of HARQ in 3GPP LTE System Level Simulation[A]. Proceedings of 2011 4th IEEE International Conference on Computer Science and Information Technology(ICCSIT 2011) VOL04[C]. 2011

[3] Sea State Parameters Extraction from Radar Images:System Design and Implementation[A]. Proceedings of 2011 IEEE CIE International Conference on Radar(RADAR 2011)[C]. 2011

[4] Design and Analysis of a Parallel Architecture for Distributed Radar Simulation[A]. Proceedings of 2011 IEEE International Conference on Computer Science and Automation Engineering(CSAE 2011) VOL03[C]. 2011

[5] A Model of Distributed Interprocess Communication System[A]. Proceedings of 2009 Second International Workshop on Knowledge Discovery and Data Mining[C]. 2009

[6] Bassem R Mahafza,Atef Z Elsherbeni.Matlab Simulations for Radar Systems Design. . 2004

[7] Hu Xiaochuan.Simulator System Design and Realization Research of Airborne Phased Array Radars. . 2003 